\title{
Entrainment of floating granules behind a barrier
}

\section{Entraînement de granules flottants derrière une barrière}

\author{
AZIN AMINI, Scientific Assistant, Laboratory of Hydraulic Constructions (LCH), Ecole Polytechnique Fédérale de Lausanne \\ (EPFL), Station 18, 1015 Lausanne, Switzerland. E-mail: azin.amini@epfl.ch (author for correspondence)
}

JEAN-LOUIS BOILLAT, (IAHR Member), Research Associate, Laboratory of Hydraulic Constructions (LCH), Ecole Polytechnique Fédérale de Lausanne (EPFL), Station 18, 1015 Lausanne, Switzerland. E-mail: jean-louis.boillat@epfl.ch

ANTON J. SCHLEISS, (IAHR Member), Professor, Laboratory of Hydraulic Constructions (LCH), Ecole Polytechnique Fédérale de Lausanne (EPFL), Station 18, 1015 Lausanne, Switzerland.E-mail: anton.schleiss@epfl.ch

\begin{abstract}
To simulate the retaining capacity of an oil barrier in an uniform flow field, experiments were carried out in a laboratory flume at Ecole Polytechnique Fédérale de Lausanne (EPFL), Laboratory of Hydraulic Constructions (LCH) by using Light Expanded Clay Aggregates (LECA) and plastic particles. It was demonstrated that under appropriate assumptions for the effects of buoyancy and gravity forces, the Shields approach is suitable to predict both the entrainment of suspended granules behind a barrier and the start of leakage underneath. The phenomenon was also simulated numerically with a multiphase model using a CFD code, Fluent, and the results were compared to those of the physical experiments. The "Eulerian model" multiphase model of FLUENT was selected to simulate the phenomenon. The numerical model successfully predicts the evolution of the slick shape behind the barrier for various flow conditions. The amount of LECA that leaked from the barrier agreed well with the experimental observations.

\section{RÉSUMÉ}

Pour simuler la capacité de fixation d'une barrière à huile dans un écoulement uniforme, des expériences ont été effectuées dans un canal de laboratoire à l'Ecole Polytechnique Fédérale de Lausanne (EPFL), Laboratoire de Constructions Hydrauliques (LCH) en utilisant des Light Expanded Clay Agrégats (LECA) et des particules en plastique. Il a été démontré que dans le cadre d'hypothèses appropriées pour les effets de flottabilité et les forces de gravité, l'approche de Shields permet de prédire tant l'entraînement de granulés en suspension derrière une barrière que le début de fuite par en dessous. Le phénomène a été également simulé numériquement avec un modèle multiphase utilisant le code de CFD Fluent, et les résultats ont été comparés à ceux des expériences physiques. Le "modèle Eulerien" multiphase de FLUENT a été sélectionné pour simuler le phénomène. Le modèle numérique prédit avec succès l'évolution de la forme de la nappe derrière la barrière pour différentes conditions d'écoulement. Le montant de LECA qui a fuit de la barrière correspondait bien avec les observations expérimentales.
\end{abstract}

Keywords: Critical shear stress, Floating granule, Interfacial shear, Multiphase model, Pollution, Retaining barrier, Shields diagram

\section{Introduction}

In the framework of a research study on contractible floating barriers to confine and recuperate oil slicks (Amini 2007, Amini et al. 2008), beside rapeseed oil also Light Expanded Clay Aggregates (LECA) and plastic, pellets were used to simulate experimentally the behavior of the oil slick behind a barrier under towing conditions. The purpose of this research is to demonstrate how the entrainment of floating granules is assessed by both a shear stress analysis and by numerical simulations. Generally, granular floating materials have to be treated as non-Newtonian fluids, whose rheological properties are complicated (Zapryanov and Tabakova 1999). Therefore, a constant viscosity cannot be attributed to a slick of granules.

The entrainment of floating granules retained by a barrier installed in a flume were observed and measured under various flow conditions. The relative flow velocities can also be simulated by towing of the barrier. An attempt was made to predict the entrainment conditions with a shear stress analysis based on the approach of Shields commonly used in sediment transport. Contrary to sediments at the channel bottom the LECA granules contained by a barrier are floating. Therefore, modifications to the Shields approach are required. The derived analytical procedure presented herein allows predicting the start of leakage of the floating granules underneath the barrier. The multiphase behavior of floating granules retained behind a barrier was then simulated numerically with a Computational Fluid Dynamic (CFD) code for various flow and towing conditions, respectively, and compared to the test results.

\section{Experimental set-up and test conditions}

Experiments were conducted in a $12 \mathrm{~cm}$ wide, $6.5 \mathrm{~m}$ long and $1.2 \mathrm{~m}$ deep laboratory flume for the test conditions specified in

Revision received June 19, 2009/Open for discussion until June 30, 2010. 
Table 1 Test conditions

\begin{tabular}{lll}
\hline Test number & $\begin{array}{l}\text { LECA volume } \\
{\left[\mathrm{m}^{3} / \mathrm{m}\right]}\end{array}$ & $\begin{array}{l}\text { Barrier draft } \\
{[\mathrm{cm}]}\end{array}$ \\
\hline LR1 & 0.02 & 10 \\
LR2 & 0.02 & 15 \\
LR3 & 0.02 & 20 \\
LR4 & 0.04 & 10 \\
LR5 & 0.04 & 15 \\
LR6 & 0.04 & 20 \\
\hline
\end{tabular}

Table 1. A flow meter of $\pm 0.1 \mathrm{l} / \mathrm{s}$ accuracy controlled the discharge. At the flume outlet a rectangular weir adjusted the water level. For all tests the water depth was fixed to $90 \mathrm{~cm}( \pm 1 \mathrm{~mm})$. The buoyant, floating granules were retained behind a rigid fixed barrier. The discharge was varied to obtain a mean flow velocity upstream of the barrier between 20 and $50 \mathrm{~cm} / \mathrm{s}$ (Amini 2007, Amini et al. 2008). The LECA granules consisted of small, floated particles of burnt clay of high porosity and a mean density of $0.71 \mathrm{t} / \mathrm{m}^{3}$. The diameter of LECA granules was in the range of 8 to $16 \mathrm{~mm}$ with a mean diameter of $12.5 \mathrm{~mm}$.

Experiments were conducted for various barrier drafts and LECA volume per unit length of the barrier (Table 1). The experimental procedure was as follows:

1. A mean flow velocity of $20 \mathrm{~cm} / \mathrm{s}$ was established upstream of the barrier.

2. A defined granule volume was poured onto the water surface upstream of the barrier forming a single layer.

3. The flow velocity was increased by small increments of $2 \mathrm{~cm} / \mathrm{s}$.

4. After conditions were stabilized:

- The volume of escaped granules was measured.

- The shape of the contained granules behind the barrier and its length and thickness at various points were recorded.

- A picture was taken.

The velocity in step 3 was increased successively until only a small volume of granules remained behind the barrier and the rate of granule leakage tended to zero.

\section{Shear stress analysis of LECA granule motion}

The Shields diagram was initially derived to characterize the entrainment of sediment particles at the bottom of open-channel flows. Particle move if the instantaneous fluid force on a particle exceeds the instantaneous resisting force. The entrainment of individual grains depends on a variety of factors, both deterministic as drag and submerged gravity forces, and random as turbulence. It is therefore difficult to establish a relationship between the shear stress and initiation of motion (van Rijn 1993). Shields (1936) proposed a relationship between the particle Reynolds number $\mathrm{R}_{*}=u_{*} D_{s} / v_{w}$ and the dimensionless critical shear stress or Shields parameter $\theta=\tau_{0} / D_{s}\left(\gamma_{s}-\gamma_{w}\right)$ to predict sediment entrainment (Yalin and Karahan 1979). Here $D_{s}=$ particle size, usually taken as $d_{50}, v_{w}=$ kinematic viscosity of water and $\gamma_{w}$ and $\gamma_{s}=$ specific weights of water (subscript $w$ ) and solid (subscript $s$ ) particles, respectively. The shear stress is $\tau_{0}=\gamma_{w} J R_{h}$ and the shear velocity $v^{*}=\left(\tau_{0} / \rho_{w}\right)^{1 / 2}=\left(g J R_{h}\right)^{1 / 2}$ where $J=$ energy line slope and $R_{h}=$ hydraulic radius.

Yalin and da Silva (2001) presented in their modified Shields diagram the Shields parameter as a function of a combined dimensionless parameter $E=\left(X^{2} / Y\right)^{1 / 3}=D_{s}\left[\left(\gamma_{s}-\gamma_{w}\right) /\left(\rho_{w} v_{w}^{2}\right)\right]^{1 / 3}$, relating the solid phase (grain density and diameter) to the liquid properties (density and viscosity) and $E$ does not change with the flow conditions. The relation $Y_{c r}=\Psi(E)$ can be explicitly expressed for $0.1<E$ as

$$
\begin{aligned}
Y_{c r}= & \Psi(E)=0.13 E^{-0.392} \exp \left(-0.015 E^{2}\right) \\
& +0.045(1-\exp (-0.068 E))
\end{aligned}
$$

This modified Shields curve is used to predict the entrainment of granules. Contrary to sediment transport, particles are floating under the water surface (Fig. 1). Hence, the gravity difference should be considered as $\left(\gamma_{s}-\gamma_{w}\right)$.

Another parameter to be modified is the shear stress $\tau$. Accumulated granules behind the barrier form a triangle with a certain longitudinal angle $\beta$. This effect is similar to an adverse sloping flow in sediment transport. The shear parameter $\tau_{0}$ modifies to

$$
\tau_{b}=k_{\beta} \tau_{0}
$$

where

$$
k_{\beta}=\frac{\sin (\phi+\beta)}{\sin \phi}
$$

with $\varphi=28^{\circ}$ as the LECA angle of repose. The angle $\beta$ was measured for each test between $10^{\circ}$ and $22^{\circ}$.

The energy line slope was calculated based on the measured head loss using the generalized Bernoulli equation (Fig. 1). The upstream depth $z_{1}$ and downstream depth $z_{2}$ were measured at the upstream slick end and $10 \mathrm{~cm}$ beyond the barrier, respectively. From Eq. (4) the global losses are

$$
h=z_{1}-z_{2}+\frac{U_{1}^{2}-U_{2}^{2}}{2 g}
$$

which is due to energy loss across the slick and local losses due to flow contraction and expansion. The head loss along the slick $h_{s}$ then is

$$
h_{s}=h-h_{n}-h_{w}
$$

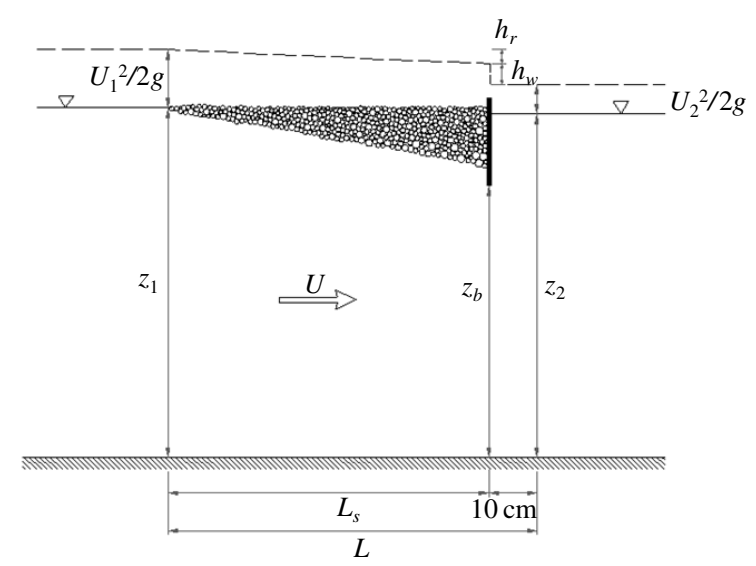

Figure 1 Scheme of LECA flume tests showing energy grade line and local losses 


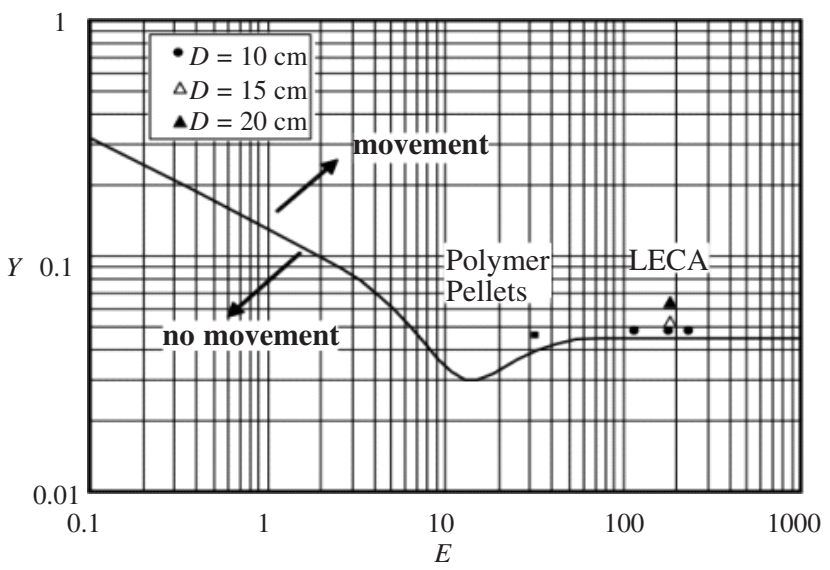

Figure 2 Modified Shields diagram, for contained material volume $V=0.02 \mathrm{~m}^{3} / \mathrm{m}$, LECA diameters $12.5,15$, and $20 \mathrm{~mm}$

where $h_{n}$ and $h_{w}$ are local head losses due to flow contraction and expansion, respectively. The head loss $h_{n}$ was assumed to be negligible. However, flow expansion may cause a significant head loss as

$$
h_{w}=\zeta_{w} \frac{U_{b}}{2 g}
$$

where $U_{b}=$ average velocity below the gate and

$$
\zeta_{w}=\left(1-\frac{A_{b}}{A_{2}}\right)
$$

where $A_{b}$ and $A_{2}$ are the flow surface below the barrier and $10 \mathrm{~cm}$ downstream of it over a total distance of $L$. The mean line energy slope along the LECA slick obtains as

$$
J=\frac{h_{s}}{L_{s}}
$$

where $h_{s}=$ head loss along the slick length $L_{s}$ (Fig. 1).

In defining a critical shear stress, the complexity is mainly caused by the lack of a clear entrainment criterion (van Rijn 1993). The initiation of motion was attributed to a frequent particle movement at various locations. The mean flow velocity upstream of the barrier was increased stepwise and the velocity at which the motion initiated was noted. To verify the application of Shields' diagram for floating granules, an additional test was undertaken involving a different value of $E=31.8$ using small polymer particles (Eraclene MP 90) with $\rho_{s}=0.96 \mathrm{gr} / \mathrm{cm}^{3}$ and $D_{s}=2.6 \mathrm{~mm}$.

The data corresponding to LECA and plastic granules for a rigid barrier of various drafts and an initial granules volume of $0.02 \mathrm{~m}^{3} / \mathrm{m}$ are shown in Fig. 2. Higher drafts are seen to increase the critical shear stress. It may be concluded that with this procedure the Shields diagram is appropriate to predict the incipient motion of floating granules behind a barrier.

\section{Numerical simulation}

\subsection{Background}

The majority of numerical models on oil spill problem simulate their spread on the water surface by applying the physiochemical spreading mechanisms and developing hindcast models (Sundararaghavan and Ertekin 1997, Yapa and Zheng 1997, 1998, Tkalich et al. 2003). Few studies have focused on the numerical modeling of oil containment process. Badesha et al. (1993) applied finite element models to study the threedimensional (3D) structural behavior of an oil spill boom. Brown et al. (1996), Goodman et al. (1996) and An et al. (1996) used the CFD code FLUENT to simulate oil-water flow in the vicinity of a containment boom.

To simulate and compare the behavior of contained LECA granules under different multiphase flow conditions, a twodimensional (2D) model was developed using FLUENT (version 6.3) (Amini et al. 2006). FLUENT uses the Finite Volume Method (FVM), a special finite difference formulation, to solve the governing equations. The most compelling feature of FVM is that the solution satisfies the conservation equations of mass, momentum, energy and phases for any control volume as well as for the entire computational domain and for any number of control volumes.

\subsection{Mesh generation and modeling conditions}

The geometrical definition and grid generation involved GAMBIT, the preprocessor bundled with FLUENT. To allow parametric studies the model was defined parametrically. For modeling fluid flow, 13,720 quadrilateral-shaped computational cells were used. The boom was modeled by two adjacent but distinct edges. A sensibility study resulted in the appropriate model for multiphase flow of LECA granules in water (see below). The turbulence was modeled using the standard $k-\varepsilon$ model. The calculations were performed with a time step of $0.001 \mathrm{~s}$ and default values of FLUENT were used for the main parameters. Detailed information on model parameters as well as the boundary conditions and turbulent dissipation rate are available (Amini 2007).

Since turbulence plays a dominant role in the transfer of e.g. mean momentum, it has to be ensured that the turbulence quantities in complex turbulent flows are properly resolved. It is therefore important that the regions where the mean flow changes rapidly and shear layers are developed, is modeled with sufficiently fine meshes. As a result, the mesh was refined in the near boom region to retain the high velocity gradient or phase volume fraction fields (Amini et al. 2006).

For the free surface, the slip wall condition was applied and the shear stress was set to zero, whereas no-slip wall condition was considered for the channel bottom and the barrier. It would be more accurate to model the free surface as an interface between water, LECA and air. However the multiphase model used can only simulate a two-phase fluid. As such the air phase was not considered in the simulations and a slip wall was applied to the upper boundary of the model. Six different models were built up, where each run represented one of the tests LR1 to LR6 (Table 1). The initial LECA volume and barrier draft were changed for each individual run according to the corresponding test. Each model was launched for three different mean flow velocities upstream of the barrier, namely 20,25 and $30 \mathrm{~cm} / \mathrm{s}$. 


\subsection{Multiphase model selection}

The transport of granular material (or bubbles and droplets) in a fluid flow is generally categorized as liquid-solid flows. There are two approaches in modeling these flows, based either on Lagrangian or Eulerian formulations. In the Lagrangian approach, the particle motion is determined by a steady Lagrangian frame, whereas in an Eulerian approach the granular phase is treated as another continuum.

With a measured maximum packing limit (maximum volume fraction) of LECA granules of $\alpha_{d} \cong 0.7$, the particulate loading is $\beta=1.7$ (Amini 2007). Considering such a high particle loading, the "general Eulerian" formulation may properly approximate the system behavior.

The "Eulerian model" is the most complex multiphase model of FLUENT. It solves the momentum and continuity equations for each phase. Coupling is achieved through the pressure and interphase exchange coefficients. The manner in which this coupling is handled depends upon the type of phases involved; granular (fluid-solid) flows are handled differently than non-granular (fluid-fluid) flows. For granular flows, the properties are obtained from the kinetic theory. The momentum exchange between the phases also depends on the mixture type modeled. The assumed parameters of the Eulerian model are specified by Amini (2007).

\subsection{Results of multiphase model}

The evolution of the slick shape during the run time of the numerical model was similar to that of the tests. The initial LECA layer over water surface accumulated behind the barrier and after a while started to escape underneath it. In general two failure modes were observed, namely entrainment and drainage failure (Amini 2007). Examples of shape evolutions for two different loss levels, i.e. failure modes, are shown in Figs. 3 and 4. In Fig. 3 the mean flow velocity is $25 \mathrm{~cm} / \mathrm{s}$, which is relatively low. After one minute a small volume of the granules escaped (loss percentage less than $50 \%$ ) due to entrainment failure. However, the mean flow velocity is higher with $35 \mathrm{~cm} / \mathrm{s}$ (Fig. 4). Therefore, the percentage of loss is more significant. After two minutes only a small volume of granules remains behind the barrier and about $80 \%$ of material is 'lost' due to drainage failure. The shape evolution in the experimental and numerical models agrees quite well, although the thickness and length of the LECA slick are not always identical.

The velocity contours and vectors in water and LECA granules are shown in Fig. 5. In Fig. 5(a) and (b) the slick shape in the test condition and simulated volume fraction of LECA for identical conditions are presented, respectively. The simulated slick shape is seen to be similar to what was observed during the test. Fig. 5(c) and (d) illustrates the contours of velocity magnitude in LECA and water, whereas Fig. 5(e) and (f) shows the velocity factors in LECA and water, respectively. The advantage of using the Eulerian multiphase model in FLUENT allows to obtain velocity contours and vectors for each phase separately. Velocity vectors show an internal circulation inside the LECA slick. The same circulation was observed during the tests. Velocity vectors in the LECA-water interfacial layer indicate high shear velocities.

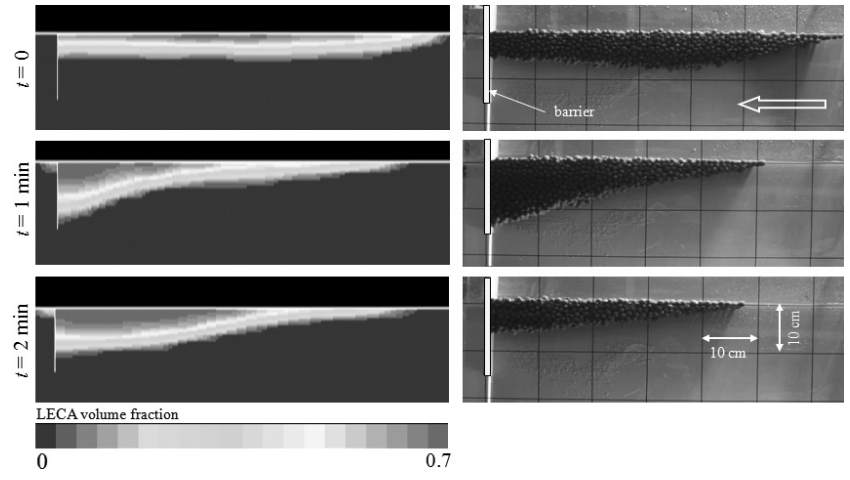

Figure 3 Evolution of LECA slick shape for numerical (left) and experimental (right) simulations, for $U=25 \mathrm{~cm} / \mathrm{s}, V=0.04 \mathrm{~m}^{3} / \mathrm{m}$, $D=15 \mathrm{~cm}$. Entrainment failure with loss percentage less than $50 \%$
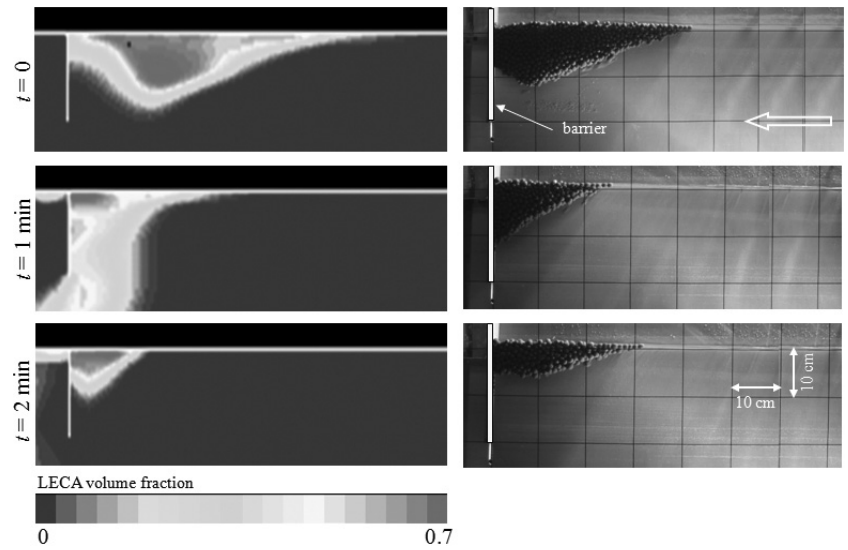

Figure 4 Evolution of LECA slick shape for numerical (left) and experimental (right) simulations, for $U=25 \mathrm{~cm} / \mathrm{s}, V=0.04 \mathrm{~m}^{3} / \mathrm{m}$, $D=20 \mathrm{~cm}$. Drainage failure with loss percentage higher than $50 \%$

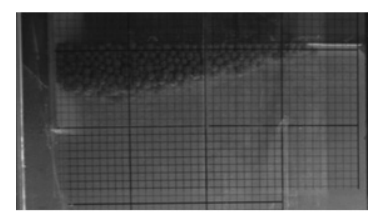

(a)

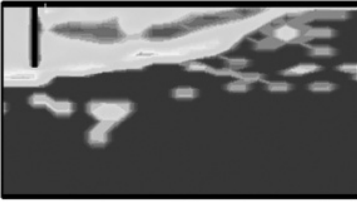

(c)

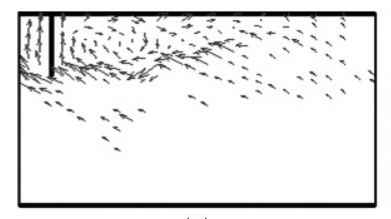

(e)

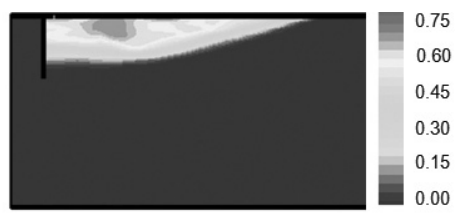

(b)

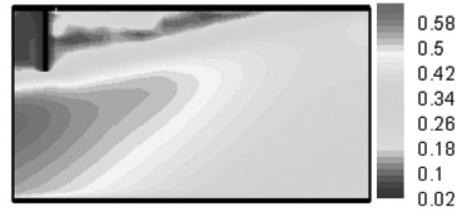

(d)

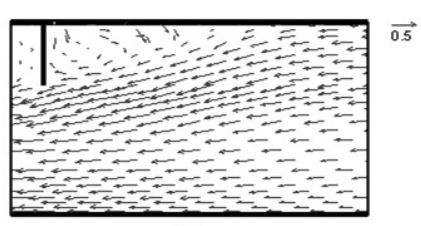

(f)
Figure 5 Detailed simulated flow characteristics for LECA slick for $U=0.35 \mathrm{~m} / \mathrm{s}, V=0.04 \mathrm{~m}^{3} / \mathrm{m}, D=20 \mathrm{~cm}$ : LECA slick shape from (a) experiments, (b) simulation; contours of velocity magnitude in (c) LECA, (d) water; velocity vectors in (e) LECA, (f) water, in [m/s]

Developing an internal User Defined Function (UDF), the volume fraction of the granular phase was determined downstream of the barrier by a loop over cells at each side of the barrier. 


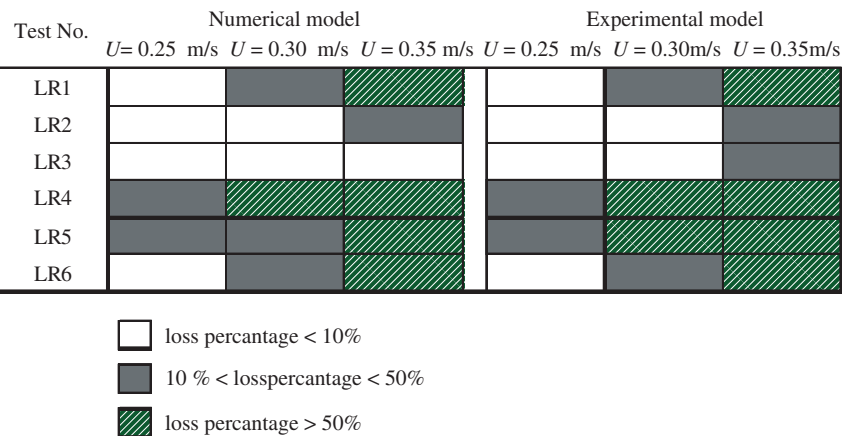

Figure 6 Comparison of LECA granule losses between numerical and experimental results for various mean velocities $U$

The amount of 'lost' LECA from the barrier was calculated from the difference between the volumes of the two sides. Figure 6 shows the LECA percentage granule loss for both numerical and experimental models at various flow velocities. The numerical model is seen to simulate the two-phase fluid behavior appropriately and the percentage of loss was in good agreement with the experiments.

\section{Conclusions}

The entrainment of floating granules contained behind a barrier placed in an uniform flow field was studied analytically and numerically, and compared with the experimental results of two different materials. The entrainment analysis was based on a modified Shields diagram. It was demonstrated that with appropriate assumptions on the effect of buoyancy and gravity forces, Shields diagram can be applied to predict the initiation of transport of floating granules.

A two-dimensional numerical model of multiphase flow was developed using FLUENT. A comparison with the experimental results confirms the capability of this code to calculate the flow characteristics of water and granules. The temporal evolution of the slick shape behind the barrier as well as the amount of material loss after initiation of motion is in good agreement with the experimental observations.

\section{Acknowledgement}

This research project was funded by the Swiss Petroleum Union under grant No. 4'09'02. The Authors thank Maziar Mahzari for his helpful comments on the numerical simulations.

\section{Notation}

$A=$ Flow area $\left(\mathrm{m}^{2}\right)$

$D=$ Draft of barrier (m)

$D_{s}=$ Typical particle size $(\mathrm{m})$

$E=$ Dimensionless combined parameter (-)

$g=$ Acceleration due to gravity $\left(\mathrm{m} / \mathrm{s}^{2}\right)$

$H=$ Global head loss $(\mathrm{m})$

$h=$ Local head loss (m)

$J=$ Energy line slope (-)

$k_{\beta}=$ Modification coefficient of shear stress (-)
$L=$ Distance $(\mathrm{m})$

$\mathrm{R}_{*}=$ Particle Reynolds number (-)

$R_{h}=$ Hydraulic radius (m)

$U=$ Flow velocity $(\mathrm{m} / \mathrm{s})$

$z=$ Water depth $(\mathrm{m})$

$\beta=$ Longitudinal angle $\left({ }^{\circ}\right)$

$\gamma=$ Specific weight $\left(\mathrm{N} / \mathrm{m}^{3}\right)$

$\theta=$ Dimensionless shear stress (-)

$\tau_{0}=$ Shear stress $\left(\mathrm{N} / \mathrm{m}^{2}\right)$

$\zeta_{w}=$ Head loss coefficient (-)

$v=$ Kinematic viscosity $\left(\mathrm{m}^{2} / \mathrm{s}\right)$

$v^{*}=$ Shear velocity $(\mathrm{m} / \mathrm{s})$

Subscripts

$1=$ Upstream of barrier

$2=$ Downstream of barrier

$b=$ Below barrier

$n=$ Narrowing

$s=$ Slick

$w=$ Widening

\section{References}

Amini, A., Bollaert, E., Boillat, J.-L., Schleiss, A. (2008). Dynamics of low-viscosity oils retained by rigid and flexible barriers. Ocean Engineering 35(14-15), 1479-1491.

Amini, A. (2007). Contractile floating barriers for confinement and recuperation of oil slicks. PhD thesis 3941. Ecole Polytechnique Fédérale de Lausanne, and Communication 35, Laboratory of Hydraulic Constructions (LCH), A. Schleiss, ed. EPFL, Lausanne.

Amini, A., Schleiss, A., Mahzari, M. (2006). Numerical modeling of floating granules containment behind a rigid barrier in a flume. 7th Intl. Conf. Hydroinformatics, Nice, France 99-106.

An, C.F., Brown, H.M., Goodman, R.H., Clavelle, E. (1996). Animation of boom failure processes. Spill Science \& Technology Bulletin 3(4), 221-224.

Badesha, S.S., Hunt, J., Wenck, E. (1993). Structural analysis of oil containment booms. 13th Intl. Conf. Oil Spill, 595-603.

Brown, H.M., Goodman, R.H., An, C.F., Bittner, J. (1996). Boom failure mechanisms: Comparison of channel experiments with computer modeling results. Spill Science \& Technology Bulletin 3(4), 217-220.

FLUENT (2007). www.fluent.com.

Goodman, R.H., Brown, H.M., An, C.F., Rowe, R.D. (1996). Dynamic modeling of oil boom failure using computational fluid dynamics. Spill Science \& Technology Bulletin 3(4), 213-216.

Sundararaghavan, H., Ertekin, R.C. (1997). Near-boom oil-slick instability criterion in viscous flows and the influence of free-surface boundary conditions. J. Energy Res. Techn. ASME, 119(1), 26-33.

Shields, A. (1936). Application of similarity principles and turbulence research to bed-load movement. Mitteilung der Preussischen Versuchsanstalt für Wasserbau und Schiffbau 26, 5-24.

Tkalich, P., Huda, K., Gin, K.Y.H. (2003). A multiphase oil spill model. J. Hydr. Res. 41(2), 1-11.

van Rijn, L.C. (1993). Principles of sediment transport in rivers, estuaries and coastal seas. Aqua Publications, Amsterdam.

Yalin, M.S., Karahan, E. (1979). Inception of sediment transport. $J$. Hydr. Div. ASCE, 105(HY11), 1433-1443.

Yalin, M.S., Ferreira da Silva, A.M. (2001). Fluvial processes. IAHR Monograph. IAHR, Delft.

Yapa, P.D., Zheng, L. (1997). Simulation of oil spill from underwater accidents 1: Model development. J. Hydr. Res. 35(5), 673-687.

Yapa, P.D., Zheng, L. (1998). Simulation of oil spill from underwater accidents 2: Model verification. J. Hydr. Res. 36(1), 117-134.

Zapryanov, Z., Tabakova, S. (1999). Dynamics of bubbles, drops and rigid particles. Kluwer Academic Publishers, Dordrecht. 